\title{
Diabetic dyslipidemia: A screening tool for cardiovascular risk assessment
}

\author{
Sadaf Ali ${ }^{1, *(\odot)}$, Nivedita L Rao ${ }^{2}$, Shiekh Amir ${ }^{3}$
}

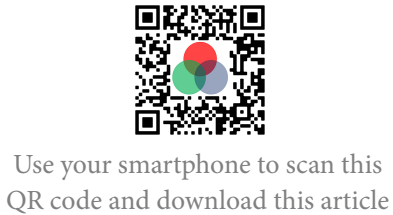

${ }^{I}$ Department of Biochemistry,

Government Medical College, Srinagar, Jammu and Kashmir, India

${ }^{2}$ Department of Biochemistry, Yenepoya Medical College, Yenepoya (deemed to be University), Mangalore, Karnataka

${ }^{3}$ Department of Forensic Medicine and Toxicology, Government Medical College, Srinagar, Jammu and Kashmir, India

\section{Correspondence}

Sadaf Ali, Department of Biochemistry, Government Medical College, Srinagar, Jammu and Kashmir, India

Email: mir.sadaf.ali@gmail.com

History

- Received: May 20, 2021

- Accepted: Nov 16, 2021

- Published: Dec 31, 2021

DOI : 10.15419/bmrat.v8i12.713

\section{Check for updates}

\section{Copyright}

(c) Biomedpress. This is an openaccess article distributed under the terms of the Creative Commons Attribution 4.0 International license.

\begin{abstract}
Introduction: Dyslipidemia is the most common threat in connection to developing cardiac disorders in type two diabetes mellitus. It has a role in the eventual development of atherosclerosis and increased mortality and morbidity. The present study was conducted to determine the lipoprotein abnormalities found in type two diabetic patients in the Mangalore region in South India. Methods: A cross-sectional study was conducted involving 95 types two diabetic patients. A fasting venous blood sample was obtained from these patients. The levels of different lipoproteins were estimated using the VITROS 5600 automatic analyzer. The patients were divided into two groups for comparison based on their duration of diabetes mellitus. The statistical tools used were the independent t-test for the comparison of the means of both quantitative variables and the Chi-square test for the proportion comparisons. The statistical package used was the Statistical Package for Social Sciences (SPSS) version 22. Results: According to the results of this study, deranged lipid profile levels were seen in most of the diabetic patients in group two. It was observed that about $67.3 \%$ of the patients in group 2 had higher serum cholesterol levels while a relatively lower number had a high level of cholesterol in group one. There was a significant ( $p$-value of $<0.001$ ) association between the two groups. There was an insignificant association between diabetes mellitus and high-density lipoprotein levels. In this study, triglyceride levels were also very high in most of the diabetic patients ( $p$-value of 0.003), further increasing the risk of obesity and metabolic syndrome. Very low-density lipoprotein and low-density lipoprotein levels were also significantly increased with a significant $p$-value. Conclusion: Most diabetic patients tend to have dyslipidemias. Screening them for dyslipidemias may be useful to prevent insulin resistance, atherosclerosis, and metabolic syndrome. Drugs like statins inhibit the rate-limiting step of cholesterol biosynthesis, leading to a lower blood cholesterol. This may help to normalize their lipid profiles and to prevent severe adverse outcomes like coronary artery disease and stroke in diabetic patients. A healthy lifestyle and dietary modifications may also help to promote good health in the patients with diabetes mellitus.
\end{abstract}

Key words: Atherosclerosis, Cholesterol, Diabetes mellitus, Dyslipidemias, High density lipoprotein, Lipid profile, Metabolic syndrome, Triglyceride

\section{INTRODUCTION}

Diabetes mellitus is a metabolic disorder that is characterized by hyperglycemia and insulin resistance. It is associated with a spectrum of other metabolic disorders $^{1}$. Dyslipidemia in both type 1 and type $2 \mathrm{DM}$ has been observed in different studies. The metabolic clearance of lipoproteins in diabetes mellitus remains disturbed. However, the relationship between diabetes mellitus and the development of atherosclerosis is not fully understood. Dyslipidemia is one of the major CVD risk factors and it plays a significant role in the pathogenesis of atherosclerosis ${ }^{1}$.India is also known as the diabetic capital of the world with a maximum number of diabetic cases ${ }^{2}$. In comparison with the general population, the prevalence of dyslipidemia is significantly higher in type 2 diabetes mellitus $^{3}$. Diabetes mellitus contributes to an interrup- tion in the metabolism of lipids, especially lipoproteins, and this leads to insulin resistance. The interrelations among obesity, insulin resistance, and hyperinsulinism contribute to the development of various complications. Risk factors such as altered insulin levels and lipid profile status are related to the fatty tissue reserves in the body. This may lead to metabolic syndrome and insulin resistance syndrome which further adds to the risk, leading to cardiovascular complications ${ }^{4}$. The treatment of diabetic dyslipidemia is essential to prevent adverse outcomes, to delay the development of secondary complications, and to ensure a healthy life ${ }^{5}$. In type 2 diabetic patients, triglycerides levels are usually high and the high-density lipoprotein cholesterol levels are low ${ }^{6}$. These lipoprotein abnormalities may be present alone or in association with other metabolic disorders ${ }^{7}$. The prevalence 
of dyslipidemia varies based on the geographic location, the type of population studied, and the level of socioeconomic development.

It has been well observed that good control of diabetes and the associated lipid levels provides excellent benefits for diabetic patients. Post-meal high blood sugar levels and high lipoproteins are risk factors for the development of vascular diseases ${ }^{1,8}$. Blood sugars and lipid abnormalities in patients can be better controlled by providing information to the patients and educating them about lifestyle modifications and the disease outcomes ${ }^{9}$. Geographical distribution, race, and culture affect specific lipid profile patterns in type 2 diabetes mellitus patients regardless of their glucose levels. The development of ethnic-specific strategies and guidelines on risk assessment and the prevention of CVD due to dyslipidemia are required ${ }^{4}$. To prevent and improve dyslipidemias in diabetes mellitus, it is important to change the individual lifestyle and to inculcate exercise and dietary changes routinely. However, pharmacological treatment such as an intake of statins and fenofibrate might also be needed to control hyperlipidemic states ${ }^{\mathbf{1}}$. A higher prevalence of increased serum cholesterol, high triacylglycerol, and high low-density cholesterol than the control group was seen among the diabetic patients, justifying the statement that diabetic patients are at a higher risk of cardiovascular disease ${ }^{3,10}$.In this study, we estimated the various lipoprotein levels in sera of type two diabetic patients in the Mangalore region of South India. It may be beneficial to study the levels of various lipoprotein abnormalities in type two diabetic patients to develop measures to prevent complications early on by prescribing lipid-lowering drugs and adopting a healthy lifestyle. Although many studies have been done on this topic, our study focuses on South Indian diabetic patients from the Dakshina Kannada region of Mangalore to find the differences accordingly. Our study also focused on the length of diabetes mellitus and the groups were made according to this duration. Not many studies have made a comparison in this manner. The duration of diabetes was emphasized as many studies suggest that an increased duration of diabetes mellitus increases the chance of developing vascular complications in diabetic patients ${ }^{11}$.

\section{METHODS}

\section{Patients}

The sample consisted of 95 diagnosed ambulatory type two diabetic patients who attended the Department of Medicine, Yenepoya Hospital for treatment.
The study was initiated after seeking ethical clearance from the Institutional Ethical Committee. The patients were considered only after informed written consent was given by them. They were ambulatory and not critically ill. Patients who were critically ill were not included in this study. The patients were grouped into two groups based on their diabetes mellitus duration.

Group 1: Patient with less than a ten-year duration of diabetes mellitus.

Group 2: Patients with more than a ten-year duration of diabetes mellitus.

An increased duration of diabetes mellitus increases the risk of the development of vascular complications in diabetic patients. Patients with a longer duration of diabetes mellitus are more prone to the development of complications. There exists a relationship between hyperlipidemia and the development of vascular complications in diabetes mellitus ${ }^{4}$. In our study, we did not use a questionnaire. A detailed clinical history from every patient was taken, blood samples were collected, and the laboratory reports of the sample patients were compiled.

\section{Study design}

Cross-sectional.

\section{Sampling Method, calculation of sample size}

The sampling method used in this study was simple random sampling. The descriptive statistics were reported using mean $\pm \mathrm{SD}$ for the continuous variables and frequency for the categorical variables. The statistical tools used were the independent $t$-test for comparison and the Chi-square test for the proportional comparisons. A p-value of $<0.05$ was determined to be statistically significant. The statistical package used was SPSS version 22. The samples were controlled by gender in the two groups, and the groups were based on the duration of diabetes mellitus.

\section{Ethical issues}

The ethical issues that were faced while conducting this study were that some of the patients were reluctant to give consent, and they were not well-educated. They were counseled and the study was explained to them. However, some of the patients did not want to participate, therefore they were excluded from this study.

\section{Time and date of study}

This study was conducted from July 2016 to July 2017 in Yenepoya University, Mangalore. 


\section{History and examination}

A detailed clinical history was taken from every patient. Demographic parameters were also compiled for each patient. Associated comorbid conditions were also taken into consideration.

\section{Inclusion and exclusion criteria}

Ambulatory type two diabetic patients were included for the purpose of this study. Patients who were critically ill and who had associated nephropathy, uncompensated heart diseases, and cancer were excluded from this study.

\section{Blood sampling and investigations}

Fasting blood samples were collected with all aseptic precautions undertaken. The blood samples were centrifuged, and the lipoproteins were estimated through the application of automated methods using the VITROS 5600 integrated dry chemistry analyzer.

\section{Total Cholesterol estimation}

The VITROS CHOL Slide is a multi-layered analytical element coated on polyester support. It is an enzymatic method (Standard operating procedures of NABL lab manual, Yenepoya University, Located at Yenepoya Central Diagnostic Laboratory). A drop of the sample is placed on the slide and it is evenly spread to the underlying layers. The Triton X-100 surfactant in the spreading layer dissociates cholesterol and its esters from the lipoprotein complexes in the sample. Cholesterol ester hydrolase hydrolyses them further, and the free cholesterol is then oxidized to form cholestenone and hydrogen peroxide in the presence of cholesterol oxidase.

\section{Triglyceride estimation}

The VITROS TRIG Slide method was performed. After spreading to the underlying layers, glycerol diffuses to the reagent layer where it is phosphorylated by glycerol kinase in the presence of adenosine triphosphate. In the presence of L- $\alpha$-glycerol-phosphate oxidase, $\mathrm{L}-\alpha$-glycerophosphate is then oxidized into dihydroxyacetone phosphate and hydrogen peroxide.

\section{HDL estimation}

The VITROS direct HDL Slide method was performed using the VITROS direct HDL Slides and the VITROS Chemistry Products Calibrator Kit 25 as part of the VITROS 5600 Integrated System. The VITROS dHDL Slide is a multilayered analytical element coated on a polyester support. The method is based on the non-HDL precipitation method, followed by enzymatic detection.

\section{LDL estimation}

The VITROS dLDL reagent is a dual-chambered package containing stable ready to use liquid reagents that are used in a two-step reaction to quantitatively measure LDLC in the first step. The addition of R1 and non-LDL cholesterol (such as HDL, VLDL, and Chylomicron) is selectively eliminated by the reaction through cholesterol esterase and cholesterol oxidase. The addition of reagent 2 initiates the second step in which the catalase is immediately inactivated with sodium azide. Surfactants then aid in the dissociation of cholesterol and cholesterol esters from the LDL particles and promote a reaction with cholesterol esterase and oxidase.

\section{Statistical analysis of the data}

The statistical tools used were the independent $t$-test for the comparison of the means of both quantitative variables, and the Chi-square test for the proportion comparisons. The statistical package used was the Statistical Package for Social Sciences (SPSS) version 22.

\section{RESULTS}

In the present study, a high level of serum cholesterol was found especially in the patients that had experienced a longer duration of diabetes mellitus. Among group 2, 67.3\% of the patients had higher serum cholesterol levels, while a relatively lower number had a high cholesterol in group 1, as shown in Table 1. This suggests that serum cholesterol levels showed statistically significant differences between the two groups with a $\mathrm{p}$-value of $<0.001$. This indicates a strong association between dyslipidemia due to high cholesterol, DM duration, and diabetic microvascular complications. Diabetes mellitus usually presents with dyslipidemias that can lead to various cardiovascular diseases in diabetic patients.

However, there was an insignificant association for DM in our study concerning HDL levels, as shown in Table 2. These results are similar to those of a study conducted by Samatha P (2012) where HDL cholesterol did not correlate well in diabetic patients. On the other hand, a significant result between DM and LDL cholesterol was obtained among the study participants, as shown in Table 3. It was found that diabetic patients tend to have a higher tendency for dyslipidemias. This may lead to the development of various cardiovascular disorders.

Similarly, Singh (2012) revealed that dyslipidemia was common in type 2 diabetic patients, especially as raised LDL levels strongly suggest the need for further investigation to relate the effects of dyslipidemia 


\begin{tabular}{|c|c|c|c|c|c|c|}
\hline & & \multicolumn{3}{|c|}{ Hypercholesterolemia } & \multirow[t]{2}{*}{ Total } & \multirow[t]{2}{*}{ p-value } \\
\hline & & Absent & Border line & Present & & \\
\hline \multirow{2}{*}{$\begin{array}{l}\text { Group } 1 \text { (Duration of diabetes } \\
\text { mellitus was }<10 \text { years) }\end{array}$} & $\mathrm{N}$ & 31 & 2 & 13 & 46 & \multirow[t]{4}{*}{$<0.001$} \\
\hline & $\%$ & $67.4 \%$ & $4.3 \%$ & $28.3 \%$ & $100.0 \%$ & \\
\hline \multirow{2}{*}{$\begin{array}{l}\text { Group } 2 \text { (Duration of diabetes } \\
\text { mellitus was }>10 \text { years) }\end{array}$} & $\mathrm{N}$ & 14 & 2 & 33 & 49 & \\
\hline & $\%$ & $28.6 \%$ & $4.1 \%$ & $67.3 \%$ & $100.0 \%$ & \\
\hline \multirow[t]{2}{*}{ Total } & $\mathrm{N}$ & 45 & 4 & 46 & 95 & \\
\hline & $\%$ & $47.4 \%$ & $4.2 \%$ & $48.4 \%$ & $100.0 \%$ & \\
\hline
\end{tabular}

All the values of lipid profile have been taken as per ATP III guidelines.

Total cholesterol (mg/dl): < 200: Desirable level; 200 - 239: Border line;

240 and above: High

Table 2: HDL status in diabetic study groups

\begin{tabular}{|c|c|c|c|c|c|c|}
\hline & & \multicolumn{3}{|c|}{ HDL levels } & \multirow[t]{2}{*}{ Total } & \multirow[t]{2}{*}{ p-value } \\
\hline & & High & Low & Normal & & \\
\hline $\begin{array}{l}\text { Group } 1 \text { (Duration of } \\
\text { diabetes mellitus was }<10\end{array}$ & $\mathrm{~N}$ & 1 & 5 & 40 & 46 & \multirow[t]{4}{*}{0.223} \\
\hline years) & $\%$ & $2.2 \%$ & $10.9 \%$ & $87.0 \%$ & $100.0 \%$ & \\
\hline $\begin{array}{l}\text { Group } 2 \text { (Duration of } \\
\text { diabetes mellitus was }>10\end{array}$ & $\mathrm{~N}$ & 1 & 12 & 36 & 49 & \\
\hline years) & $\%$ & $2.0 \%$ & $24.5 \%$ & $73.5 \%$ & $100.0 \%$ & \\
\hline \multirow[t]{2}{*}{ Total } & $\mathrm{N}$ & 2 & 17 & 76 & 95 & \\
\hline & $\%$ & $2.1 \%$ & $17.9 \%$ & $80.0 \%$ & $100.0 \%$ & \\
\hline
\end{tabular}

HDL (mg/dl): < 40: Low; > 60: High

Table 3: LDL status in diabetic study groups

\begin{tabular}{lcccccc}
\hline & \multicolumn{3}{c}{ LDL status } & Total & p-value \\
Group 1 (Duration of diabetes & $\mathrm{N}$ & 22 & 1 & 23 & 46 & 0.029 \\
mellitus was < 10 years) & $\%$ & $47.8 \%$ & $2.2 \%$ & $50.0 \%$ & $100.0 \%$ & \\
Group 2 (Duration of diabetes & $\mathrm{N}$ & 36 & 0 & 13 & 49 \\
mellitus was > 10 years) & $\%$ & $73.5 \%$ & $0.0 \%$ & $26.5 \%$ & $100.0 \%$ \\
\hline Total & $\mathrm{N}$ & 58 & 1 & 36 & 95 \\
& $\%$ & $61.1 \%$ & $1.1 \%$ & $37.9 \%$ & $100.0 \%$ \\
\hline
\end{tabular}

LDL (mg/dl): < 100: Optimal; 100 - 129: Near optimal; 130 - 159: Borderline high; 160 - 189: High; 190 and above: Very high

and the abnormalities of insulin resistance in type 2 diabetics.

VLDL status in the diabetic study groups showed that the patients in group two had significantly higher VLDL levels with a significant $p$-value of $<0.001$, as shown in Table 4. Therefore, it was observed that among the diabetic patients of our study, patients with an increased duration of diabetes mellitus had significantly higher VLDL levels.
In this study, triglyceride levels were also remarkably high in most diabetic patients, as shown in Table 5. The values were suggestive of significant results. The increased triglyceride levels increase the risk for obesity and metabolic syndrome.

The overall lipid profiles in the present study showed there to be a positive correlation between DM and total cholesterol, Triglycerides, LDL, and VLDL, suggesting that there are significant dyslipidemias in di- 


\begin{tabular}{lccccc}
\hline Table 4: VLDL status in diabetic study groups & \multicolumn{2}{c}{ VLDL status } & Total & p-value \\
\hline & \multicolumn{7}{c}{ High } & Normal & & \\
Group 1 (Duration of diabetes & $\mathrm{N}$ & 13 & 33 & 46 & $<0.001$ \\
mellitus was $<10$ years) & $\%$ & $28.3 \%$ & $71.7 \%$ & $100.0 \%$ & \\
& $\mathrm{~N}$ & 32 & 17 & 49 & \\
Group 2 (Duration of diabetes & $\%$ & $65.3 \%$ & $34.7 \%$ & $100.0 \%$ & \\
mellitus was $>10$ years & $\mathrm{N}$ & 45 & 50 & 95 & \\
\hline Total & $\%$ & $47.4 \%$ & $52.6 \%$ & $100.0 \%$ & \\
\hline
\end{tabular}

Table 5: Prevalence of hypertriglyceridemia in diabetic study groups

\begin{tabular}{lcccccc}
\hline & \multicolumn{7}{c}{ Hypertriglyceridemia } & Total & p-value \\
& & Absent & Border line & Present & & 0.003 \\
$\begin{array}{l}\text { Group 1 (Duration of diabetes } \\
\text { mellitus was < 10 years) }\end{array}$ & $\mathrm{N}$ & 32 & 1 & 13 & 46 & $100.0 \%$ \\
Group 2 (Duration of diabetes & $\%$ & $69.6 \%$ & $2.2 \%$ & $28.3 \%$ & 49 \\
mellitus was > 10 years & $\mathrm{N}$ & 17 & 1 & 31 & $100.0 \%$ \\
\hline Total & $\%$ & $34.7 \%$ & $2.0 \%$ & $63.3 \%$ & 95 \\
& $\mathrm{~N}$ & 49 & 2 & 44 & $100.0 \%$ \\
\hline
\end{tabular}

ATPIII classification of serum Triglycerides (mg/dl): < 150: Normal; 150 - 199: Border line high; 200 - 499: High; 500 or above: Very high

abetic patients. The study groups showed a strong and significant relation concerning the total cholesterol, LDL, VLDL, and triglyceride values. Therefore, it becomes essential to screen diabetic patients for dyslipidemias earlier to prevent cardiovascular diseases. Longer-term diabetes mellitus makes these patients prone to developing more dyslipidemias, increasing the development of atherosclerosis and the subsequent risk of cardiovascular and cerebrovascular events.

\section{DISCUSSION}

In the present study, the main finding was that a strong association exists between dyslipidemia due to high cholesterol levels and the increased duration of diabetes mellitus. Most of our study participants had increased total cholesterol, triglycerides, VLDL, and LDL cholesterol levels.

Previously, a study conducted by Bhaghaya S. K (2017) revealed that the levels of triglycerides, total cholesterol, and LDL cholesterol were higher in cases in comparison to the controls in patients with diabetes mellitus, suggesting that dyslipidemias are present in diabetic patients ${ }^{7,12}$. Similarly, Singh (2012) revealed that in type 2 diabetic patients, dyslipidemias espe- cially were found to be among the raised LDL levels frequently found. It may be suggested to investigate these patients and to correlate the consequences of dyslipidemia and insulin resistance in type 2 diabetes mellitus patients ${ }^{4}$. Similar results were obtained by the study conducted by Deepa Singh (2015). They showed considerably lower good cholesterol levels in the study population and significant dyslipidemia in diabetic patients, making them more prone to developing cerebrovascular and cardiovascular accidents. Shivan and K.G (2012) conducted a study in India and concluded that dyslipidemia in diabetic patients resulted in a low level of good cholesterol. Mumtaz Ali Sheikh conducted a study in 2010. The study results showed a deranged lipid profile in most diabetic patients. In different studies, type 2 diabetes mellitus patients are at a higher risk of cardiovascular disease development ${ }^{3,4,13}$. The results for HDL cholesterol were similar to the results of a study conducted by Samatha P (2012), where HDL cholesterol did not correlate well in diabetic patients.

Quantifying the atherogenic lipoproteins is the best determinant of cardiovascular risk ${ }^{8}$. The concentrations of these particles are the best way to go about risk prediction and it can also help to determine the 
best therapeutic goals for dyslipidemias. Sterols, especially cholesterol, enter the artery wall and are internalized by macrophages, forming foam cells and the associated inflammatory process ${ }^{10,12}$. Foam cells are the histological diagnostic marker of atherosclerosis 8 . Thus, the only way sterols get into the artery wall is through the lipoprotein particles transporting them. These particles are driven into the arterial intima by concentration gradients. Therefore, optimum levels of these lipoproteins in the blood will help to prevent deleterious health hazards in diabetic patients and the general population. The early detection of hyperlipidemia and the treatment of the same may be helpful to prevent the development of CAD.

One of the major risk factors for cardiovascular disease in type 2 diabetes mellitus is dyslipidemia ${ }^{1,5,14}$. It plays an essential role in the pathogenesis and progression of micro and macrovascular complications in such patients ${ }^{15}$. Diabetes mellitus increases the deposition of lipids through numerous biochemical derangements in the metabolic pathways. The early diagnosis and treatment of dyslipidemia is the main cornerstone in the prevention of its multiple complications ${ }^{5}$. Patients with T2DM are at a higher risk of developing vascular diseases because of lipoprotein derangements ${ }^{\mathbf{1 6}}$. Obesity contributes mainly to type 2 diabetes mellitus. Nearly $80 \%$ of diabetic patients are usually overweight or obese ${ }^{\mathbf{5}}$. Type 2 diabetes mellitus can be prevented by maintaining healthy lifestyle behaviors ${ }^{9}$. The lipid metabolism is affected in diabetes mellitus, substantiated by the fact that all of the lipid fractions were increased in diabetic patients compared to the healthy controls. So hyperlipidemia is quite a common finding in connection to diabetes mellitus ${ }^{4,7}$.

Diabetic female patients with a higher BMI of $\geq 30$ are predisposed to have more lipoprotein abnormalities ${ }^{17}$.The results for body mass index (BMI) and blood pressure were found to be significantly higher in women compared to men of the same age, and they were exposed more strongly to risk factors like atherogenic dyslipidemia and higher BMI and BP compared to men ${ }^{18}$.

The main limitation of this study was that more patients could have been involved, and we could compare the results with those of neighboring geographical areas.

\section{CONCLUSIONS}

Lipoproteins are the key molecules that, if deranged, can lead to cardiovascular diseases. This has been more frequently seen in patients with type two diabetes mellitus. More often, type two diabetic patients tend to have dyslipidemias, especially hypercholesterolemia. Overall, the lipid profiles in the present study showed there to be a positive correlation between diabetes mellitus and the total cholesterol, Triglycerides, LDL, and VLDL levels suggestive of significant dyslipidemia in diabetic patients. The study groups showed there to be a strong and significant relation concerning the total cholesterol, LDL, VLDL, and triglyceride values. Screening diabetic patients early becomes essential to prevent cardiovascular disease and to intervene at a relatively earlier stage. Prescribing drugs like statins and fenofibrate may prove helpful in these patients as the former drug affects the cholesterol rate-limiting step. Later on, it will become useful to decrease the triglyceride levels. Lifestyle changes and maintaining the same are also useful in these patients, and they can help to prevent dyslipidemia and subsequent coronary artery disease development.

\section{ABBREVIATIONS}

BMI: Body mass index, BP: Blood pressure, HDL: High density lipoprotein, LDL: Low density lipoprotein, VLDL: Very low-density lipoprotein

\section{ACKNOWLEDGMENTS}

The authors of this study thank Dr. Ravi Vaswani, Professor, Department of General Medicine, Yenepoya Medical College Hospital for helping in patient selection for the study. The authors also thank Ms. Megha, statistician at Yenepoya (deemed to be University) for conducting statistical analysis of data.

\section{AUTHOR'S CONTRIBUTIONS}

All the authors made substantial contributions to conception, design, acquisition of data, analysis, and interpretation of data; took part in drafting the article, revising it critically for the intellectual content; approving the final version for publishing and agree to be accountable for all aspects of the work. All authors read and approved the final manuscript.

\section{FUNDING}

None.

\section{AVAILABILITY OF DATA AND MATERIALS}

Data and materials used and/or analyzed during the current study are available from the corresponding author on reasonable request. 


\section{ETHICS APPROVAL AND CONSENT TO PARTICIPATE}

The study was conducted in accordance with the amended declaration of Helsinki. The Institutional Ethical Committee of Yenepoya University approved this study, and all the study participants gave their informed written consent.

\section{CONSENT FOR PUBLICATION}

Not applicable.

\section{COMPETING INTERESTS}

The authors declare that they have no competing interests.

\section{REFERENCES}

1. Bhagyashree KB. Lipid profile in Diabetes Mellitus. Journal of Exercise Science and Physiotherapy. 2012;8(1):7-10.

2. International Diabetes Federation. IDF Diabetes atlas. 9th edBrussels, Belgium: IDF 2019. http://www.diabetesatlas.org/; 2019.

3. Shigematsu E, Yamakawa T, Kadonosono K, Terauchi Y. Effect of sitagliptin on lipid profile in patients with type 2 diabetes mellitus. Journal of Clinical Medicine Research. 2014;6(5):32735. PMID: 25110536. Available from: 10.14740/jocmr1889w.

4. Singh G, Kumar AK. A Study of Lipid Profile in Type 2 Diabetic Punjabi Population. Journal of Exercise Science and Physiotherapy. 2012;8(1):7-10. Available from: 10.18376//2012/v8i1/ 67599.

5. Nyasatu GC, Elichilia RS, Tolbert S, Isaack A. Lyaruu. Lipid Profile of Type 2 Diabetic Patients at a Tertiary Hospital in Tanzania: Cross Sectional Study. Journal of Endocrinology and Diabetes. 2017;4(1):1-6. Available from: 10.15226/2374-6890/ 4/1/00170.

6. Samatha P, Venkateswarlu M, Prabodh S. Lipid Profile Levels in Type 2 Diabetes Mellitus from the Tribal Population of Adilabad in Andhra Pradesh, India. Journal of Clinical and Diagnostic Research. 2012;6(4):593-596.
7. Shivanand KG, Manjunath ML, Jeganathan PS. Lipid profile and its complications in diabetes mellitus. International Journal of Biomedical and Advance Research. 2012;3(10):775-80. Available from: 10.7439/ijbar.v3i10.767.

8. Mumtaz AS, Santosh K, Rafi AG. Type 2 Diabetes Mellitus and Lipid Abnormalities. Jlumhs. 2010;9(3):145-7. Available from: https://lumhs.edu.pk/jlumhs/Vol09No03/pdfs/v9n3oa05.pdf.

9. Michael $S$, et al. Obesity and Diabetes. AADE Practice Synopsis. 2014;2014:1-7.

10. Nosheen A. Influence of glycation on low density lipoprotein in diabetic cardiovascular disease patients. E3 J. Biotechnol Pharm Res. 2013;4(5):81-8.

11. Zoungas S, Woodward M, Li Q, Cooper ME, Hamet P, Harrap S, et al. Impact of age, age at diagnosis and duration of diabetes on the risk of macrovascular and microvascular complications and death in type 2 diabetes. Diabetologia. 2014;57(12):246574. PMID: 25226881. Available from: 10.1007/s00125-0143369-7.

12. Kissebah A, Schectman G. Polyunsaturated and saturated fat, cholesterol, and fatty acid supplementation. Diabetes Care. 1988;11(2):129-42. PMID: 3289863. Available from: 10.2337/ diacare.11.2.129.

13. Rajendra DB, Kamal L. Types of Dyslipidemia in Type 2 DM Patients of Bhubaneswar region. Annals of Clinical Chemistry and Laboratory Medicine. 2015;1(1):33-6.

14. Mohammad IK, Fair U, Javaid M, Zahid K. Hyperlipedemia in Diabetes Mellitus. Journal of Postgraduate Medical Institute; 18(2):261-268.

15. Deepa S, Arvinder S, Rakesh K, Islam K. Dyslipidemia in Type 2 Diabetes Mellitus Patients of Udaipur Population. Sch J App Med Sci. 2015;3:813-5.

16. Shubhangi AK, Krunal KT, Anu NG, Meenakshi K, Rajani RA. Thyroid dysfunction in patients with type 2 diabetes mellitus at tertiary care centre. National Journal of Medical Research. 2013;3(4):377-80.

17. Sandra DB, Dawn S, Ruth DL. Partnering with diabetes educators to improve patient outcomes. Diabetes Metab Syndr Obes. 2014;7:45-53. PMID: 24550679. Available from: 10 . 2147/DMSO.S40036.

18. Rademacher ER, Jacobs DR, Moran A, Steinberger J, Prineas RJ, Sinaiko A. Relation of blood pressure and body mass index during childhood to cardiovascular risk factor levels in young adults. Journal of Hypertension. 2009;27(9):1766-74. PMID: 19633567. Available from: 10.1097/HJH.0b013e32832e8cfa. 
Ready to submit your manuscript? Choose Biomedpress and benefit from:

- Fast, convenient online submission

- Through peer-review by experienced researchers

- Rapid publication on acceptance

- Free of charge (without publication fees)

Learn more http://www.biomedpress.org/journals/
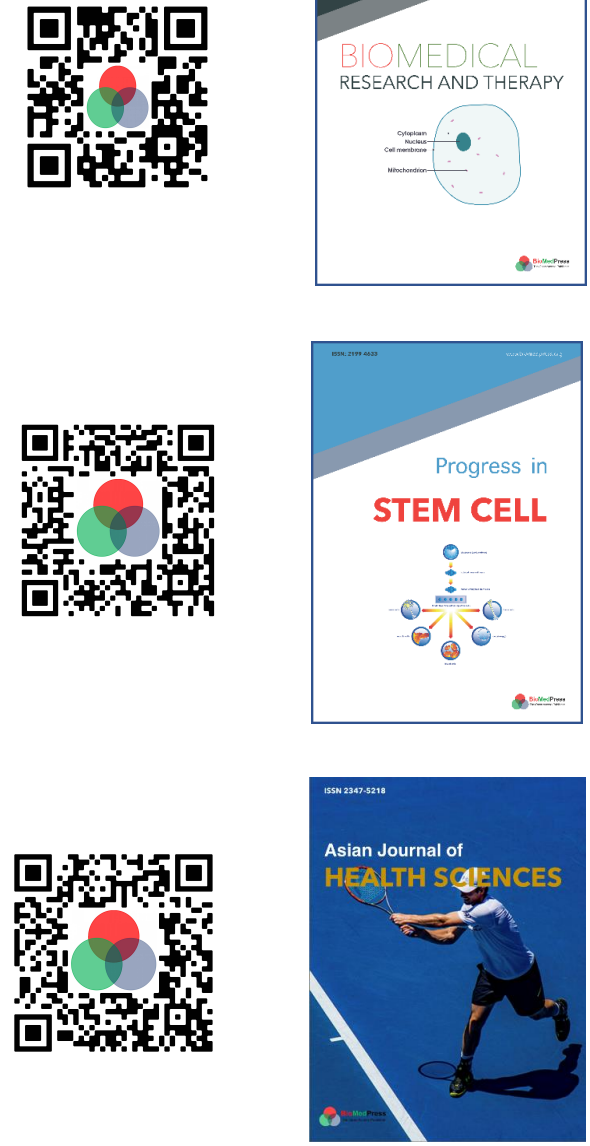

Asian Journal of Health Sciences

ISSN: 2347-5218

Indexed: Google Scholar

Acceptance Rate (2020): 72.89\%

Article Publishing Charge: Free

Submission to first editorial decision: 16.5 days

Biotechnological Research

ISSN: 2395-6763

Indexed: Google Scholar

Acceptance Rate (2020): $67.02 \%$

Article Publishing Charge: Free

Submission to first editorial decision: 28.5 days 